\title{
La subsidiariedad de la Cláusula Antielusiva General ante la escisión corporativa regulada en el numeral 3 del artículo 105 de la Ley del Impuesto a la Renta en Perú
}

\section{The subsidiarity of the General Anti Avoidance Rule before the corporate split regulated on the third numeral of the 105th article of the Income Tax Law}

Gonzalo Alonso Escalante Alpaca

Universidad Católica San Pablo, Arequipa, Perú • gaescalante@ucsp.edu.pe

\section{Resumen}

Existen discrepancias doctrinales respecto a la relación que existe entre la Cláusula Antielusiva General (CAG) y las Cláusulas Antielusivas Específicas (CAE), ya que se considera que la relación puede ser de exclusión, de subsidiariedad o mixta. Este artículo analiza las tres posturas respecto a las CAE recogidas en el numeral 3 del artículo 105 de la Ley del Impuesto a la Renta (LIR), donde se regula una presunción absoluta de generación de renta gravada y distribución de dividendos, cuando los sujetos que perciben acciones como consecuencia de una escisión transfieran o cancelen dichas acciones en una posterior reorganización, siempre que se cumplan con los parámetros cuantitativos y temporales previstos en dicha norma. Para el análisis se considera la normativa tributaria y los comentarios de la doctrina especializada. En conclusión, existe una relación mixta que da lugar a una aplicación subsidiaria de la $\mathrm{CAG}$ respecto a la referida $\mathrm{CAE}$, siendo que una posición en contrario implicaría tener por válido el hecho de que los contribuyentes puedan tergiversar los parámetros cuantitativos y temporales recogidos en la CAE para poder evitar la configuración de las consecuencias impositivas previstas en esta.

Palabras clave: cláusula antielusiva general (CAG), cláusula antielusiva específica (CAE), impuesto a la renta (IR), relación mixta, subsidiariedad. 


\begin{abstract}
There are several differences in the doctrine regarding the relation that exists between the General Anti-Avoidance Rule (GAAR) and the Specific Anti-Avoidance Rules (SAARs), since it is considered that such relation can be of exclusion, subsidiarity or a mixed of both. This article analyses all three positions regarding the SAARs regulated on the third numeral of the 105th article of the Income Tax Law (ITL), where it is stipulated an absolute presumption according to which there is an income generated as well as a benefits distribution if the subjects that perceived equities from a corporate split transfer or cancel such equities because of a further corporate reorganization, as long as the quantitative and temporary parameters considered on this regulation are fulfilled. For the current analysis, the tax law and the specialized doctrine have been considered, which has allowed us to conclude that on the case of the previously mentioned regulation there is a mixed relation that allows a subsidiary application of the GAAR regarding such SAARs, since recognizing an opposite position will imply that it is valid for the taxpayers to distort the quantitative and temporary parameters contained on the SAARs to avoid the configuration of its taxable consequences.
\end{abstract}

Keywords: general anti-avoidance rule (GAAR), specific anti-avoidance rule (SAAR), income tax (IT), mixed relation, subsidiarity.

\title{
1. Introducción
}

La elusión fiscal en Perú, entendida como el desarrollo de prácticas para ocultar total o parcialmente la configuración del hecho imponible, tiene como consecuencia directa la erosión de la base imponible en el Perú, afectando así la caja fiscal. Ello implica que nuestro Estado se vea privado de los necesarios recursos para poder cumplir con sus objetivos y suplir las necesidades de los ciudadanos, causando la reducción de la capacidad de gasto público. Por ello, resulta muy importante conocer cuáles son los mecanismos recogidos en la normativa tributaria para combatir el desarrollo de prácticas elusivas.

Las normas recogidas en nuestro ordenamiento para combatir la elusión fiscal son principalmente de dos tipos, la CAG y las CAE, por lo que se debe conocer la forma en la que ambas se relacionan para mitigar el riesgo de prácti- cas elusivas por parte de los contribuyentes, sobre todo mediante el uso de estructuras de planeamiento tributario donde se involucran reorganizaciones empresariales.

El numeral 2 del artículo 104 de la LIR dispone que dentro de las opciones con las que cuenta una empresa al momento de realizar un procedimiento de reorganización empresarial está el realizar una revaluación voluntaria, en la que la diferencia entre el mayor valor pactado y el costo computable no estará gravada con el IR, siempre que no se realice la distribución de dicha diferencia a favor de los accionistas. Se señala además que el mayor valor atribuido con motivo de la revaluación voluntaria no tendrá efecto tributario alguno para determinar el costo computable ni la base de depreciación de los bienes transferidos con motivo de la reorganización empresarial. 
Ahora bien, el numeral 3 del artículo 105 del mismo cuerpo normativo recoge una presunción absoluta conforme a la cual se considera que la ganancia regulada en el numeral 2 del artículo 104 de la LIR, constituida por la diferencia entre el costo computable de los activos transferidos y el monto de la revaluación voluntaria, es distribuida cuando una empresa realice una escisión y las acciones o participaciones recibidas por los socios, como consecuencia de dicho procedimiento, sean transferidas en propiedad o canceladas por una posterior reorganización empresarial, siempre que se presenten de forma concurrente las siguientes condiciones:

- Las acciones o participaciones transferidas o canceladas representen más del $50 \%$ en capital o derechos de voto del total de acciones o participaciones emitidas a los socios de la empresa escindida.

- La transferencia o cancelación de las acciones o participaciones se realice hasta el cierre del ejercicio siguiente a aquel en que entró en vigencia la escisión. De ser el caso que la transferencia o cancelación se realice en distintas oportunidades, se presumirá a su vez que la distribución se hizo efectiva al momento en que la transferencia o cancelación superó el 50\% previamente mencionado.

Se dispone, además, que ante estas circunstancias se genera una renta gravada en la sociedad que adquirió el bloque patrimonial como consecuencia de la escisión.

Así pues, tenemos que la regulación del numeral 3 del artículo 105 de la LIR se configura como una $\mathrm{CAE}$, que tiene por objeto crear consecuencias impositivas respecto al hecho de que los contribuyentes pretendan utilizar la escisión con el objetivo de anular o reducir la carga impositiva aplicable a las transferencias de activos que se encubran bajo la figura de una escisión corporativa. Este es el caso de una presunción absoluta, respecto a la cual no cabe la posibilidad de actuar prueba en contrario.

Por su parte, la CAG está recogida dentro de la Norma XVI del Título Preliminar del Código Tributario (CT), donde se dispone que la Administración Tributaria cuenta con la facultad de realizar la recalificación de una transacción que se considera elusiva, con el fin de aplicar las consecuencias fiscales que resulten atribuibles, siempre que del análisis de dicha transacción se obtenga que se han superado los test de propiedad y de sustancia económica.

En tal sentido, es necesario analizar cuál es la vinculación entre la referida $\mathrm{CAG}$ y las previamente mencionadas $\mathrm{CAE}$, para efecto de determinar si existe la posibilidad de reconocer una relación mixta entre ambas figuras, lo que permite la aplicación subsidiaria de la CAG cuando se evidencie que se ha distorsionado la figura de la escisión con el objetivo de evitar la aplicación de las CAE, pero se ha mantenido como objetivo el anular o reducir la carga impositiva aplicable a una transferencia de activos a través de la figura del fraude a la ley.

No se ha identificado algún estudio que analice, en forma específica, la relación existente entre la CAG y las CAE recogida en el numeral 3 del artículo 105 de la LIR, centrándose en la subsidiariedad como criterio rector de dicha relación.

En el análisis efectuado, nuestra hipótesis considera que la relación entre la CAG y las 
CAE mencionada es mixta y ello permite a su vez reconocer la subsidiariedad de la $\mathrm{CAG}$, siendo que, en caso de que un contribuyente distorsione una operación de forma tal que esta no calce con lo dispuesto en las CAE, corresponderá que se analice la transacción conforme a los parámetros previstos dentro de la CAG para determinar si la operación califica o no como una práctica elusiva.

Así pues, se desarrolla una investigación jurídico-propositiva sobre la base de la CAG y su relación con la CAE consagrada en el numeral 3 del artículo 105 de la LIR. Se utiliza además el método hipotético-deductivo para establecer si la hipótesis planteada dentro del artículo resulta cierta o no.

El análisis está dividido en tres apartados: el primero versa sobre la regulación aplicable a la CAG y las CAE en el ordenamiento peruano; el segundo apartado versa sobre la relación entre la CAG y las CAE desde un punto de vista doctrinal; $\mathrm{y}$, por último, el tercer apartado recoge el análisis efectuado de la vinculación existente entre la CAG y la CAE recogida dentro del numeral 3 del artículo 105 de la LIR.

\section{La Cláusula Antielusiva General y las Cláusulas Antielusivas Específicas en el ordenamiento peruano}

\subsection{El problema de la elusión tributaria}

La elusión tributaria es un fenómeno presente en Perú y que ha generado un importante daño en la recaudación de recursos fiscales en nuestro Estado, como consecuencia de la erosión de la base imponible mediante el desarrollo de diversas estructuras que tienen por objetivo ocultar o reducir los efectos impositivos de las transacciones llevadas a cabo. Esto ha derivado en una pérdida de recursos fiscales, que resultan sumamente necesarios en una coyuntura postpandemia, donde la caja fiscal se encuentra afectada por la paralización económica, consecuencia de la emergencia sanitaria.

Dentro de las diversas posibilidades empleadas para configurar prácticas elusivas, está el que los contribuyentes suelen recurrir a las estructuras de reorganización empresarial, a través de las cuales se utilizan normas societarias con el fin de obtener un indebido ahorro tributario.

Las respuestas del ordenamiento tributario peruano son diversas para enfrentar a este tipo de prácticas, pero se pueden dividir entre la CAG y las CAE, ya que ambas han sido introducidas en nuestra legislación para combatir, con un mayor o menor rango de aplicación, como se explicará más adelante, la elusión fiscal de manera efectiva. Es necesario entender la forma en que estas normas se aplican y los alcances de cada una de ellas, así como la vinculación existente entre ambos tipos de normas, bajo la premisa de que ambas tienen por objetivo principal el combatir la elusión fiscal.

\subsection{Fraude a la ley y la economía de opción}

Respecto al fraude a la ley, Tarsitano indica:

El fraude a la ley constituye una categoría tendiente a sancionar aquellas conductas que, sin ejercer violencia en la naturaleza y en la forma de los actos y negocios jurídicos que le sirven de apoyo, terminan por producir un resultado práctico contrario a los fines que el derecho protege. Es este el medio con el que el derecho reacciona contra las conductas no queridas, que contradicen los intereses tutelados por las normas y podrían verse violados bajo una lectura 
instrumental estricta de aquellos actos y negocios. El acto privado, «pese a salvar las apariencias y respetar la letra de la ley, logra violar su precepto en espíritu [...] configurándose una violación indirecta, que mientras respeta la letra de la norma, desvirtúa su finalidad o elude, utilizando un instrumento legal en contra del destino que le es propio». (Tarsitano, 2021).

Continúa el mismo autor señalando lo siguiente:

En la descripción de la conducta elusiva adoptamos la tesis objetiva, que encuentra en el fraude a la ley una reacción del derecho al uso instrumental de un negocio que se hace servir para una ilicitud indirecta, y es ajena a esta valoración jurídica la mala o la buena conciencia del otorgante, que debería quedar relegada a un motivo individual sin relevancia calificadora, en particular cuando los pasos del interesado pudieron estar guiados por el propósito de evitar el impuesto por una opción lícita. (Tarsitano, 2021).

Por su parte, Queralt, Lozano y Tejerizo señalan lo siguiente:

El fraude de ley exige la presencia de dos tipos de normas. Una es la norma (o normas) bajo cuyo amparo se realizan los actos en fraude, denominada norma de cobertura; y otra es la norma (o normas) cuya vulneración se persigue, conocida como norma defraudada. En ocasiones la norma de cobertura resulta ser, paradójicamente, la ausencia de normas que regulen el acto o actos realizados.

Así pues, de acuerdo con esta línea doctrinal, que compartimos, se puede definir el fraude de ley tributaria como el resultado contrario a una norma tributaria provocado por uno o varios actos que se han realizado bajo el amparo formal de una ley que ha sido dictada con una finalidad distinta de la perseguida por los interesados. (Queralt, Lozano y Tejerizo, 2020).

En este extremo nos remitimos a los señalado por Bravo, quien indica que:

Una de las formas de eludir la aplicación de una norma tributaria es a través de la realización de negocios anómalos en fraude a la ley tributaria. Por lo tanto, el fraude a la ley tributaria es una forma de elusión fiscal. Como lo hemos dicho en anterior oportunidad, el fraude a la ley tributaria consiste en intentar pagar el menor tributo posible mediante la realización de actos concretos artificiosos, que se intentan apoyar en una norma jurídica civil, laboral, comercial o en una combinación de ellas (normas de cobertura) para evitar la aplicación de una norma tributaria (norma defraudada), más adecuada al caso por la causa y por la finalidad económica y jurídica del hecho efectivamente realizado. A través de actos jurídicos civil, comercial o laboralmente anómalos, se busca evitar la aplicación de una norma tributaria, pretendiendo caracterizar dicho acto como uno sometido a otra norma tributaria - normalmente menos gravosa que la que en rigor corresponde-, o como uno no sometido a norma tributaria alguna. (Bravo Cucci, 2020).

Sobre la base de los textos citados se considera que el fraude a la ley se configura entonces cuando un contribuyente utiliza una norma de cobertura, que forma parte del ordenamiento jurídico, para reducir o anular la carga imposi- 
tiva aplicable, mediante el ocultamiento de los objetivos reales de la transacción efectuada. Se entiende el fraude a la ley como uno de los mecanismos a los que se recurre para eludir la norma tributaria, a través del cual se utiliza el mismo ordenamiento con el fin de limitar el impacto impositivo atribuible a un caso concreto.

En lo que se refiere a la economía de opción, el Decreto Supremo 145-2019-EF la define de la siguiente manera:

A la acción de elegir y el resultado de elegir llevar a cabo actos que tributariamente son menos onerosos que otros posibles o disponibles en el ordenamiento jurídico y respecto de los cuales no se presentan ninguna de las circunstancias previstas en los literales a) y b) del tercer párrafo de la norma XVI del Título Preliminar del Código Tributario. (MEF, 2019).

La citada norma, a su vez, proscribe la aplicación de la CAG recogida en la Norma XVI del Título Preliminar del CT respecto a los actos que se adecuen al concepto de economía de opción.

Por su parte, Bravo indica respecto a la economía de opción que:

Se trata de operaciones realizadas utilizando el ordenamiento jurídico del país, optando entre las posibilidades que en él se permiten, a los efectos de realizar hechos no gravados o sometidos a algún beneficio tributario. El planeamiento tributario que utilice una economía de opción supone un actuar lícito. (Bravo Cucci, 2020).

Sobre este concepto Queralt, Lozano, Tejerizo y Casado refieren que:
La economía de opción, aceptada por el Derecho, permite que un determinado contribuyente acomode su actuación a la forma que le resulte menos gravosa, de entre todas las que ofrece el ordenamiento (por ejemplo, para llevar a cabo una actividad conjuntamente con otras personas, se podrá optar entre constituir una sociedad anónima o una comunidad de bienes), pero, y ésta es la diferencia con el fraude a la ley, la forma empleada no ha de resultar anómala en relación con el fin perseguido por las partes. La economía de opción es lícita siempre que no entrañe un abuso de las formas jurídicas; y siempre que no se recurra al empleo de fórmulas jurídicas atípicas en relación con los fines que pretenden conseguirse. (Queralt, Lozano y Tejerizo, 2020).

En este orden de ideas, Sánchez indica que:

Con relación a la economía de opción. En el fraude de ley, a diferencia de la economía de opción, el ordenamiento quiere una tributación o una mayor tributación a la pretendida. Se afirma que [...] en la elusión, a diferencia de lo que sucede en la economía de opción, el ordenamiento jurídico quiere que la tributación tenga lugar aunque se utilicen actos o negocio jurídicos válidos no contemplados formalmente en la norma como presupuestos de imposición. STS de 24 de febrero de 2014 (rec. de cas. 1347/2011) (Sánchez Huete, 2019).

Picón, a su vez, define la economía de opción conforme al siguiente detalle:

Existe economía de opción cuando las relaciones privadas o empresariales se 
organizan de tal forma que no debe satisfacerse impuesto alguno o solo alguno de reducida cuantía, sin que para ello - a diferencia del caso en que se evita la realización del hecho imponible - haya que renunciar a la conducta querida o a una administración económica razonable. En este caso no se entra en conflicto ni con la letra ni con el sentido de la ley. (Picón Gonzales, 2020).

Sobre la base de las definiciones citadas respecto a la configuración de la economía de opción, resulta necesario para establecer la existencia o no de una transacción que califique como tal, que se analice si optar por una opción transaccional resulta lícito, normal y no significa en modo alguno el aprovechamiento indebido de una norma de cobertura con el fin de obtener un ahorro fiscal indebido, lo que implicaría la existencia de un fraude a la ley.

A su vez debemos considerar que la regulación de la economía de opción en nuestro ordenamiento se hace en base a la aplicación de la CAG más no de las CAE. Por lo tanto, la determinación del hecho de que una transacción se encuentra dentro de los parámetros de la economía de opción y no se constituye como un fraude a la ley, se configura como una salvaguarda de la misma únicamente respecto de la CAG. En lo que se refiere a las CAE el criterio para establecer que una transacción no se configura como elusiva será únicamente el incumplimiento de los preceptos de las CAE correspondientes.

\subsection{CAG en el ordenamiento tributario}

Referirnos a la CAG es introducirnos en un ámbito que genera mucha discusión a nivel doctrinal, ya sea que se le considere como una norma de naturaleza material o si se le considera más bien como una norma de naturaleza procedimental, o incluso si es que se analiza la necesidad o pertinencia de su inclusión en nuestro ordenamiento tributario, ya que esta norma resulta de las más controvertidas con las que se cuenta en el ámbito fiscal. Así pues, la CAG resulta una de las instituciones que ha generado más discusión y respecto a la cual se han realizado análisis de los más profundos para efecto de estudiar determinados aspectos de la misma.

El presente apartado se centra en el análisis de las principales características de la CAG y más adelante se comentarán las razones que podrían justificar, o justifican, que se haya insertado una norma de tales rasgos dentro de la normativa fiscal peruana.

La inclusión dentro del ordenamiento peruano de la CAG no ha carecido de escollos. En 1996 hubo un primer intento de contar con una $\mathrm{CAG}$ dentro del texto de la Norma VIII del Título Preliminar del CT aprobado mediante Decreto Legislativo 816, sin embargo, se derogó al poco tiempo de su entrada en vigencia. Luego se publicó el 19 de julio de 2012 el Decreto Legislativo 1121, a través del cual se incluye dentro del ordenamiento fiscal la Norma XVI del Título Preliminar del CT, cuyo texto es el siguiente:

Para determinar la verdadera naturaleza del hecho imponible, la SUNAT tomará en cuenta los actos, situaciones y relaciones económicas que efectivamente realicen, persigan o establezcan los deudores tributarios.

En caso que se detecten supuestos de elusión de normas tributarias, la 
Superintendencia Nacional de Aduanas y Administración Tributaria - SUNAT se encuentra facultada para exigir la deuda tributaria o disminuir el importe de los saldos o créditos a favor, pérdidas tributarias, créditos por tributos o eliminar la ventaja tributaria, sin perjuicio de la restitución de los montos que hubieran sido devueltos indebidamente.

Cuando se evite total o parcialmente la realización del hecho imponible o se reduzca la base imponible o la deuda tributaria, o se obtengan saldos o créditos a favor, pérdidas tributarias o créditos por tributos mediante actos respecto de los que se presenten en forma concurrente las siguientes circunstancias, sustentadas por la SUNAT:

a) Que individualmente o de forma conjunta sean artificiosos o impropios para la consecución del resultado obtenido.

b) Que de su utilización resulten efectos jurídicos o económicos, distintos del ahorro o ventaja tributarios, que sean iguales o similares a los que se hubieran obtenido con los actos usuales o propios.

La SUNAT, aplicará la norma que hubiera correspondido a los actos usuales o propios, ejecutando lo señalado en el segundo párrafo, según sea el caso.

Para tal efecto, se entiende por créditos por tributos el saldo a favor del exportador, el reintegro tributario, recuperación anticipada del Impuesto General a las Ventas e Impuesto de Promoción Municipal, devolución definitiva del Impuesto General a las Ventas e Impuesto de Promoción Municipal, restitución de derechos aran- celarios y cualquier otro concepto similar establecido en las normas tributarias que no constituyan pagos indebidos o en exceso.

En caso de actos simulados calificados por la SUNAT según lo dispuesto en el primer párrafo de la presente norma, se aplicará la norma tributaria correspondiente, atendiendo a los actos efectivamente realizados. (Texto Único Ordenado del Código Tributario aprobado mediante Decreto Supremo 133-2013-EF, 2013).

Conforme se obtiene de la norma citada, el texto de la $\mathrm{CAG}$, recogida en los párrafos segundo al quinto del artículo XVI del Título Preliminar del CT, dispone la aplicación de dos test o exámenes que deben ser superados por una determinada operación, para que la recalificación resulte aplicable por parte de la Administración Tributaria.

Respecto a estos test, Mur señala que:

En este orden de ideas, los incisos a) y b) de la Norma XVI vienen a configurar un set de dos evaluaciones que deben realizarse para establecer si los actos constituyen o no elusión, siendo imprescindible que el producto de ambas evaluaciones lo confirme.

- El primero de ellos es un test de «causalidad», cuyo propósito es acreditar que el acto o los actos realizados van o no en línea con sus fines, esto es, si los actos corresponden o no a sus fines o si los fines carecen de importancia, en cuyo caso son pura forma.

- Segundo, es un test de «suficiencia», cuyo objeto es confirmar o no que el único 
propósito de los actos es tributario, pues las otras ventajas económicas o jurídicas que resultan de los actos indebidos también se podrían alcanzar a través de la realización de actos típicos. (Mur Valdivia, 2018).

Por nuestra parte, proponemos la siguiente explicación sobre los mencionados test:

- Primer examen: El test de propiedad, que responde a identificar si la transacción efectuada resulta anómala respecto a los efectos jurídicos obtenidos a través de esta. Se debe identificar si la forma en la que se ha estructurado la transacción tiene como objetivo ulterior la consecución de un resultado, al cual se hubiera podido acceder mediante una forma regular, normal y directa.

- Segundo examen: El test de sustancia económica u objetivo de negocios, a través del cual se debe analizar si la transacción llevada a cabo ha tenido una finalidad sustentada en buscar un eficiente desarrollo económico de las operaciones del contribuyente o si esta se basa únicamente en la obtención de un ahorro fiscal.

En lo que respecta a la manipulación artificiosa de la estructura del negocio, Caliendo refiere que:

La relación del hecho económico, negocio jurídico y efecto tributario deben guardar una correlación de coherencia. Así, no es posible que un hecho económico que genera renta, formalizado a través de un contrato de compraventa, no sea tributado, cuando haya ocurrido el evento, dado que cuando sea vertido en un lenguaje competente se torna en un hecho jurídico que se adecua a la hipótesis de incidencia de la norma jurídico-tributaria e implica necesariamente el surgimiento de la obligación tributaria. (Caliendo, 2018).

Así las cosas, cuando una transacción cumple con los test previstos en la CAG resultará aplicable la recalificación del acto y la consecuente imputación de las consecuencias fiscales atribuibles. Para su aplicación se deberán considerar las disposiciones previstas en el artículo 62-C del CT, que no es materia de este artículo.

Asimismo, como consecuencia de la aplicación de la CAG se considerará configurada la infracción prevista en el numeral 9 del artículo 178 del mismo cuerpo normativo.

\subsection{CAG y la capacidad contributiva}

El Tribunal Constitucional, en la sentencia recaída en el expediente STC 2727-2002-AA/ TC, señala sobre la capacidad contributiva que:

Asimismo, (el principio de no confiscatoriedad) se encuentra directamente conectado con el derecho de igualdad en materia tributaria o, lo que es lo mismo, con el principio de capacidad contributiva, según el cual, el reparto de los tributos ha de realizarse de forma tal que se trate igual a los iguales y desigual a los desiguales, por lo que las cargas tributarias han de recaer, en principio, donde exista riqueza que pueda ser gravada, lo que evidentemente implica que se tenga en consideración la capacidad personal o patrimonial de los contribuyentes. (Sentencia del Tribunal Constitucional 2727-2002-AA/TC, 2002).

Respecto a la constitucionalidad del principio de capacidad económica, Gamba menciona lo siguiente: 
El artículo 74 de la CP no reconoce «expresamente» como un principio aplicable en materia tributaria, el de «capacidad económica», situación que no debe llevarnos a afirmar que este no resulte exigible, pues, su contenido puede derivarse sin mayores dificultades del «principio de igualdad». Por esta razón, la falta de un reconocimiento expreso del principio de capacidad económica en el artículo 74 de la $\mathrm{CP}$, no puede llevarnos al absurdo de considerar que este no tiene plena vigencia en nuestro ordenamiento tributario o que resulta un principio disponible por el legislador. Por el contrario, consideramos que tan inconstitucional resulta una norma que vulnera los principios de igualdad o no confiscatoriedad - reconocidos expresamente por el texto constitucional-, como aquella norma que no satisface las exigencias que impone el principio de capacidad económica — derivado del principio de igualdad-. Además de ello, han sido múltiples los casos en los que nuestro Tribunal Constitucional (TC) ha obtenido efectos prácticos importantes derivados del principio de capacidad económica, al momento de efectuar el control de la constitucionalidad de las leyes tributarias (vía acciones de inconstitucionalidad), así como al pronunciarse sobre casos concretos (vía acciones de garantía). (Gamba Valega, 2014).

Sobre la relación entre la capacidad contributiva y la igualdad, Spisso refiere que:

Íntimamente vinculado e integrado con el principio de igualdad, se halla el principio de capacidad contributiva, que para Ferreiro Lapatza, más que un tercer principio es una forma de entender la generalidad y la igualdad; un modo de aplicarlas que se halla hoy universalmente consagrado.

El que todos deban contribuir al sostenimiento de los gastos del Estado debe ser entendido, en un Estado democrático, como que los llamados a contribuir son aquellos que tengan capacidad económica para soportar la carga, y en forma proporcional y progresiva a dicha capacidad. (Spisso, 2011).

Ahora bien, entendemos que existe la necesidad de recoger la CAG dentro del ordenamiento peruano en aras de dotar a la Administración Tributaria de una herramienta adicional para poder combatir la elusión fiscal. El combate a la elusión fiscal encuentra respaldo constitucional en el principio de igualdad recogido en el artículo 74 de la Constitución, en cuyo amparo se debe gravar de forma igual a quienes se encuentren en las mismas condiciones y gravar de manera distinta a quienes se encuentran en una diferente situación respecto a un determinado hecho imponible. De este principio de igualdad surge un subprincipio de rango constitucional que es la capacidad contributiva, entendida como el mandato al legislador tributario de gravar únicamente los hechos o actos que impliquen la exteriorización de una determinada capacidad económica, así como la obligación de los contribuyentes de tributar conforme a la exteriorización de riqueza que manifiesten y sobre la cual se configure un hecho imponible. La exteriorización de riqueza se puede dar mediante la obtención de renta, el consumo o el patrimonio.

La CAG es, pues, un elemento primordial para que en nuestro sistema se otorgue un tratamiento igualitario a quienes exterioricen 
una misma capacidad contributiva, haciendo que los sujetos que recurrieron a prácticas elusivas, con el fin de obtener un ahorro fiscal, sean susceptibles de ser sometidos a las cargas impositivas que les resulten aplicables por los actos cuyos efectos tributarios pretendieron ocultar o reducir.

Es una prerrogativa del Estado velar porque los contribuyentes tributen conforme a su real capacidad contributiva, con la aplicación de un tratamiento igualitario. La CAG, según se indicó previamente, no es sino una herramienta para la aplicación de dicha prerrogativa, que puede recalificar los actos elusivos llevados a cabo por los contribuyentes, adjudicar a estos su real carga impositiva y aplicar las multas que resulten pertinentes.

\subsection{Las CAE en el ordenamiento tributario}

Las CAE han sido incluidas dentro de nuestro ordenamiento con el objetivo de combatir prácticas elusivas independientes, identificadas por parte de la Administración Tributaria, respecto de los contribuyentes que las realizan con el objetivo de obtener un ahorro fiscal aprovechando un vacío normativo.

Las CAE han generado que se amplíen los supuestos sujetos a gravamen, que atribuyen consecuencias impositivas respecto a circunstancias que, antes de la inclusión de las CAE dentro del ordenamiento tributario, no se encontraban afectas a gravamen alguno como consecuencia de una falta de regulación.

Se configuran, pues, como una respuesta ante el abuso del derecho que realizaban los contribuyentes para evitar las consecuencias fiscales de las transacciones llevadas a cabo o reducir su efecto impositivo. Esta respuesta se ha estructurado a través de diversas normas que han permitido combatir la elusión mediante ficciones donde no existe conexión lógica entre el hecho comprobado y la consecuencia fiscal atribuible; así como a través de las presunciones relativas o absolutas en las que existe una vinculación lógica entre el hecho verificado y la consecuencia impositiva imputada.

Como ejemplo de las CAE como ficciones, tenemos la aplicación de las reglas de precios de transferencia para realizar el ajuste correspondiente cuando partes vinculadas trancen sin aplicar el valor de mercado, o también el desconocimiento del gasto proveniente de países o territorios no cooperantes o de baja o nula imposición. En lo que se refiere a la aplicación de presunciones existen las relativas, como la que regula la presunción de incremento patrimonial no justificado, donde se permite la actuación de pruebas en contrario para evitar la aplicación de dicha presunción. Y en lo que respecta a las presunciones absolutas se tiene como ejemplo la recogida en el numeral 3 del artículo 105 de la LIR, conforme a la cual se presume, sin admitir prueba en contrario, la generación de renta empresarial y una distribución de dividendos en caso se violen los parámetros previstos dentro de dicha norma y que se analizarán más adelante dentro del presente artículo.

\section{Relación entre la CAG y las CAE}

\subsection{Teorías que regulan la relación entre la CAG y las CAE}

Existen en la doctrina tres formas distintas de conceptualizar la relación existente entre la CAG y las CAE, estas son la exclusión, la subsidiariedad y la mixta. En las líneas siguientes analizamos la posición doctrinal aplicable a cada una de dichas posturas: 
- Relación de exclusión:

Este tipo de relación significa, en términos resumidos, que cuando se verifique que una transacción no cumple con las disposiciones recogidas dentro de la CAE no resultará procedente analizar si la CAG es aplicable a la misma.

Chumacero indica al respecto que:

Este tipo de relación implica que, si una conducta no se subsume dentro del supuesto del hecho de la CAE, no califica como elusiva, por lo que ya no podrá ser calificada como elusiva bajo el parámetro de la CAG.

Uno de los fundamentos de este tipo de relación, se encuentra en la teoría general del derecho. Así, constituye un principio del derecho que la norma especial prevalece sobre la norma general. [...]

Otro fundamento importante que respalda este tipo de relación, se encuentra vinculado con el respeto del principio de seguridad jurídica. En efecto, dicho principio que es pilar de todo ordenamiento jurídico, procura garantizar que las reglas de juego se encuentren claras y, de esa manera, se evite la incertidumbre jurídica. (Chumacero Quispe, 2018).

Conforme a la relación de exclusión, la aplicación de la CAG se encuentra proscrita cuando se verifica, en primer término, que las disposiciones de las CAE no pueden ser utilizadas como un mecanismo para combatir la elusión en un caso concreto. Bajo este escenario, un contribuyente que pretenda realizar una práctica elusiva, deberá verificar únicamente que no se han configurado las disposiciones de las CAE, pues la aplicación de la CAG para revisar la estructura planteada no resulta factible.

- Relación de subsidiariedad:

Esta relación implica reconocer que la CAG puede aplicarse de forma irrestricta siempre que la Administración Tributaria verifique que no se encuentran presentes los elementos necesarios para calificar a una transacción como elusiva conforme al texto de una determinada CAE, permitiendo que el fisco analice si en el caso concreto resulta factible o no la aplicación de la $\mathrm{CAG}$ respecto de la misma operación. Al amparo del reconocimiento de este tipo de relación, el posterior análisis dentro de los parámetros de la $\mathrm{CAG}$ es aplicable en todo caso, incluso si se ha verificado que no se ha constituido una práctica elusiva conforme a la CAE correspondiente. 
En este punto, Lizárraga señala lo siguiente:

Teniendo en consideración que la voluntad del legislador es darle siempre a la Administración Tributaria una herramienta que le permita combatir la elusión fiscal, la CAG siempre podrá ser aplicable, incluso a los supuestos que son cubiertos por las CAEs.

La razón detrás de esta postura radica en que las normas específicas no pueden ser obstáculos sino más bien herramientas de la lucha contra la elusión fiscal, por lo que bajo esta posición se privilegia el no abuso del derecho, el principio de igualdad y el deber de contribuir.

Bajo esta posición, la CAG podrá ser aplicada por la Administración Tributaria incluso en aquellos supuestos donde existen CAEs. (Lizárraga Ibáñez, 2018).

Considerando la relación de subsidiariedad, los contribuyentes no podrán resguardarse en la no trasgresión de las reglas previstas en las CAE para realizar prácticas elusivas, pues resultará procedente la aplicación de la $\mathrm{CAG}$ como un mecanismo residual para combatir la configuración de este tipo de prácticas, incluso cuando no se hayan configurado los elementos de las CAE. Esta posición permite al fisco realizar la recalificación de los actos que pudieran considerarse como elusivos conforme a la CAG sin limitación alguna.

\section{- Relación mixta:}

Este tipo de relación prevé que la exclusión y subsidiariedad resultan aplicables respecto a determinadas CAE, dependiendo si esta ha sido incluida dentro de nuestro ordenamiento como una ficción, en cuyo caso la relación es de exclusión, o como una presunción en cuyo caso la relación es de subsidiariedad.

Al respecto Lizárraga refiere que:

Bajo esta posición la relación entre la CAG y las CAEs no dependerá de la forma en que las CAEs se encuentran configuradas. Así, si las CAEs se encuentran configuradas bajo la forma de una ficción, no podrá aplicarse la CAG, pues al haber creado el legislador una verdad jurídica ésta no puede ser desconocida por la administración tributaria.

En cambio, si la CAE se ha configurado mediante la forma de presunciones, ya sean estas relativas o absolutas, la administración tributaria podría aplicar la $\mathrm{CAG}$, ya que las presunciones se desarrollan en el ámbito probatorio de la consecuencia de un hecho conocido pero no crean una verdad jurídica material sino solo formal, mediante la relevación de la carga de la prueba de una de las partes (García Novoa 2004: página 270-271). (Lizárraga Ibáñez, 2018).

Así las cosas, tenemos que, según esta posición, la aplicación subsidiaria de la CAG respecto a una estructura empresarial que no cumple con los requisitos para que las CAE puedan ser utilizadas como mecanismo antielusivo se encontrará supeditada a la naturaleza de la CAE. Dependerá entonces si estamos ante una presunción, en cuyo caso la CAG resultará aplicable subsidiariamente, o si nos encontramos ante una CAE que tiene la naturaleza de ficción, en cuyo caso la aplicación de la CAG se encuentra proscrita; este es el caso de una relación de exclusión. 
Ampliando lo antes mencionado, se puede considerar que las presunciones recogidas en el ordenamiento tributario peruano son de dos clases. Por un lado, se tienen las presunciones absolutas, respecto a las cuales no cabe la posibilidad de presentar prueba alguna en contrario; y por otro lado están las presunciones relativas, sobre las cuales cabe la posibilidad de presentar prueba en contrario por parte de los contribuyentes, respecto a los cuales se realiza la imputación correspondiente para la aplicación de la presunción.

\section{Vinculación entre la CAG y las CAE recogida en el numeral 3 del artículo 105 de la LIR}

\subsection{La CAE prevista en el numeral 3 del artículo 105 de la LIR}

Para entender esta CAE debemos remitirnos en primer término al numeral 2 del artículo 104 de la LIR. Dicha norma reviste el siguiente texto:

Artículo 104.- Tratándose de reorganización de sociedades o empresas, las partes intervinientes podrán optar, en forma excluyente, por cualquiera de los siguientes regímenes:

\section{$[\ldots]$}

2. Si las sociedades o empresas acordaran la revaluación voluntaria de sus activos, la diferencia entre el mayor valor pactado y el costo computable determinado de acuerdo con el Decreto Legislativo $\mathrm{N}^{\mathrm{o}} 797$ y normas reglamentarias no estará gravado con el Impuesto a la Renta, siempre que no se distribuya. En este caso, el mayor valor atribuido con motivo de la revaluación voluntaria no tendrá efecto tributario. En tal sentido, no será considerado para efecto de determinar el costo computable de los bienes ni su depreciación. (Texto Único Ordenado de la Ley del Impuesto a la Renta. Decreto Supremo 179-2004-EF, 2004).

La reorganización empresarial regulada en el citado numeral puede darse mediante la fusión, la escisión o la reorganización simple. En este caso se establece que las empresas sometidas a alguno de estos procedimientos de reorganización podrán optar por realizar una revaluación voluntaria de sus activos, únicamente para efectos de la reorganización sin que ello tenga efecto fiscal alguno. La nulidad del efecto fiscal se mantendrá, en tanto no se efectúe distribución alguna de la diferencia existente entre el costo computable de los activos antes de la reorganización y el mayor valor otorgado a dichos activos como consecuencia de esta.

Comentando este numeral, Medrano refiere que:

El legislador ha querido permitir que las empresas sinceren el valor de los bienes de su activo, sin que ello dé lugar a consecuencias en el impuesto a la renta; es decir, exactamente lo que en cualquier momento puede hacer una sociedad, aun cuando ello no ocurra dentro de un proceso de reorganización. Al respecto, debe recordarse que la propia LIR, en el inciso $b$ de su artículo 24A, considera como dividendos: La distribución del mayor valor atribuido por revaluación de activos, ya sea en efectivo o en especie, salvo en títulos de propia emisión representativos del capital. (Medrano, 2018).

Ahora bien, tenemos que el numeral 3 del artículo 105 de la LIR recoge el siguiente texto: 
Artículo 105.- En el caso previsto en el numeral 2 del artículo anterior, si la ganancia es distribuida en efectivo o en especie por la sociedad o empresa que la haya generado, se considerará renta gravada en dicha sociedad o empresa.

Se presume, sin admitir prueba en contrario, que la ganancia a que se refiere el numeral 2 del artículo 104 de esta ley es distribuida en cualquiera de los siguientes supuestos:

\section{$(\ldots)$}

3. Cuando una sociedad o empresa realice una escisión y las acciones o participaciones que reciban sus socios o accionistas como consecuencia de la reorganización sean transferidas en propiedad o canceladas por una posterior reorganización, siempre que:

a) Las acciones o participaciones transferidas o canceladas representen más del cincuenta por ciento (50\%), en capital o en derechos de voto, del total de acciones o participaciones que fueron emitidas a los socios o accionistas de la sociedad o empresa escindida como consecuencia de la reorganización; y,

b) La transferencia o cancelación de las acciones o participaciones se realice hasta el cierre del ejercicio siguiente a aquél en que entró en vigencia la escisión. De realizarse la transferencia o cancelación de las acciones en distintas oportunidades, se presume que la distribución se efectuó en el momento en que se realizó la transferencia o cancelación con la cual se superó el cincuenta por ciento $(50 \%)$ antes señalado.

En el supuesto previsto en este numeral, se presume que la ganancia es distribuida por la sociedad o empresa que adquirió el bloque patrimonial escindido, y por tanto, renta gravada de dicha sociedad o empresa. Lo previsto en este párrafo se aplicará sin perjuicio del impuesto que grave a los socios o accionistas por la transferencia de sus acciones o participaciones. (Texto Único Ordenado de la Ley del Impuesto a la Renta. Decreto Supremo 179-2004-EF., 2004).

Sobre este numeral, Medrano ha mencionado que:

Se supone que la ganancia es distribuida por la sociedad que adquirió el bloque patrimonial escindido y la renta se imputa al ejercicio en que se efectuó la distribución. El hecho de pagar el impuesto no faculta a considerar como costo el mayor valor atribuido por revaluación.

Al respecto, cabe señalar que las consecuencias referidas solo se precipitan si la transferencia versa sobre las acciones que se emitieron a los socios de la escindida a raíz de la reorganización. Por lo tanto, si el bloque patrimonial se transfiere a una sociedad ya existente, no habría incidencia alguna si las acciones que se enajenan son las que los socios tenían en dicha sociedad antes de la operación. (Medrano, 2018).

En el caso de la CAE citada nos encontramos ante una presunción absoluta, respecto a la cual no cabe la posibilidad de presentar prueba en contrario. Ampliando la definición de presunción absoluta, Saavedra indica lo siguiente:

La presunción legal absoluta o denominada también iure et de iure o de Derecho y por Derecho, tienen como característica que no admiten prueba en contrario, respecto 
del hecho presunto; es decir, una vez establecida por ley, opera de pleno derecho. La única forma de destruir la presunción absoluta es, a través del establecimiento de medios probatorios que evidencien la inexistencia del hecho cierto que constituye el soporte del hecho presunto. La carga de la prueba en este tipo de presunciones la tiene quien se beneficia de la presunción, pues tendrá a cargo la obligación de probar el hecho cierto o hecho base. (Saavedra Rodríguez, 2019).

Las consecuencias de la aplicación de la CAE son entonces que, en primer término, se presuma una distribución de dividendos por la diferencia existente entre el costo computable de los activos transferidos vía escisión y su valor luego de la revaluación voluntaria, y a su vez, que se presuma la existencia de renta gravada en la empresa beneficiaria de la transferencia de activos como consecuencia de dicha reorganización.

\subsection{La relación mixta y la subsidiariedad de la CAG respecto a la CAE recogida en el numeral 3 del artículo 105 de la LIR}

Como mencionamos en el numeral precedente, la presunción del numeral 3 del artículo 105 de la LIR es absoluta, ya que no admite prueba en contrario. Ante este escenario, nos cuestionamos cuál es la relación que debe reconocerse entre esta CAE y la CAG.

Para el desarrollo de nuestro análisis, proponemos un ejemplo que se encuentra fuera del ámbito de aplicación de la CAE, para poder determinar la relación existente con la CAG.

En el caso hipotético que la empresa C (cuyos accionistas son A y B) escinda todos sus activos a favor de la empresa $\mathrm{D}$, excepto un inmueble que tiene la intención de transferir a favor de la empresa E. Una vez efectuada la escisión, los accionistas de C (A y B) proceden a realizar la transferencia de las acciones de dicha empresa a favor de la empresa E, con lo que se cumple el objetivo primigenio de la estructuración realizada, que implica transferir el inmueble que quedó como único activo de C, luego de la escisión, a favor de la empresa E, utilizando para ello la norma societaria de cobertura que regula la transferencia de acciones, con un gravamen efectivo por concepto del IR del $5 \%$ sobre la diferencia entre el costo computable y el valor de venta de las acciones. Se genera pues un ahorro importante, considerando que la tasa del IR de Tercera Categoría, que resultaría aplicable si la venta del inmueble se realizase directamente por la empresa $\mathrm{C}$ a favor de $\mathrm{E}$, es del $29.5 \%$ (régimen general) sobre la renta neta. 
La estructura propuesta en el ejemplo se esquematiza en los siguientes pasos:

Paso 1: Escisión de los activos de la compañía $\mathrm{C}$ a favor de la empresa $\mathrm{D}$, dejando como único activo de la sociedad escindida el inmueble que se pretende transferir.

\section{Figura 1}

\section{Escisión de los activos}

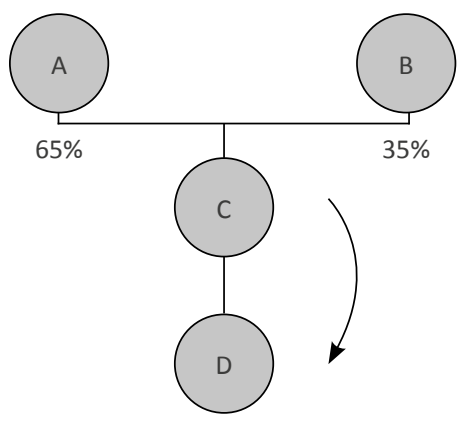

C escinde todo su patrimonio a D excepto un inmueble

Nota. Elaboración propia.

Paso 2: Se realiza la enajenación de las acciones de la empresa $\mathrm{C}$ a favor de la empresa $\mathrm{E}$, estando la renta obtenida sujeta a un gravamen por concepto de IR con una tasa del 5\%.

\section{Figura 2}

Enajenación de las acciones

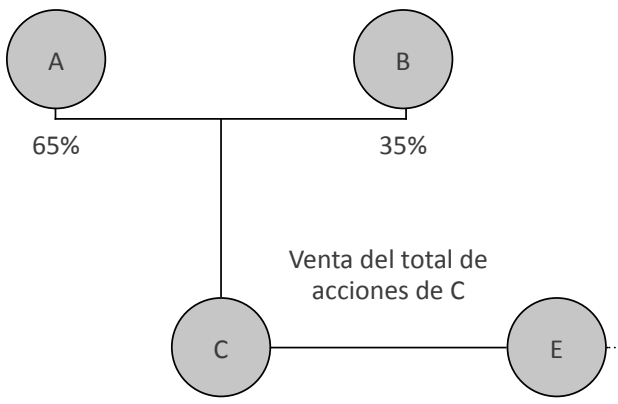

¿Resulta aplicable la CAG? 
Los accionistas de $\mathrm{C}$ saben del interés de la empresa $\mathrm{E}$ de adquirir uno de los activos de la primera. Asimismo, estos accionistas conocen que el costo tributario es considerablemente más elevado si la venta se realiza directamente por C (tasa del IR del 29.5\%), a si se realiza una venta de acciones (tasa del IR del 5\%). Para ello han estructurado una venta de acciones, evitando caer dentro del supuesto antielusivo recogido en el numeral 3 del artículo 105 de la LIR, en tanto las acciones transferidas son las que siempre fueron propiedad de los accionistas A y B, mas no las acciones que se adquieren como consecuencia de la escisión, haciendo inaplicable la CAE mencionada.

Se ha de reconocer entonces una relación mixta entre las CAE y la CAG que permitirá a la Administración Tributaria, subsidiariamente, aplicar la CAG para determinar si en este caso existe o no una práctica elusiva que tenga como fin principal la obtención de un ahorro fiscal indebido. Ello considerando que la CAE se regula bajo una presunción absoluta. La aplicación subsidiaria de la CAG va a permitir que se combata efectivamente la intención elusiva, base sobre la cual se ha estructurado la transacción.

En aplicación subsidiaria de la CAG es necesario realizar los dos test, para efecto de determinar si la práctica efectuada califica o no como elusiva conforme a sus parámetros. Ello conforme al siguiente detalle:

- Test de propiedad: El efecto jurídico perseguido con la transferencia de las acciones de $\mathrm{C}$ es trasladar la propiedad del inmueble que quedó como su único activo. La transferencia de acciones no supera el test al haberse utilizado una operación anómala para encubrir la venta de un activo.
- Test de sustancia económica: En este caso, no existe objetivo económico además de realizar la transferencia de un activo. Sin embargo, se cubre este fin mediante una transferencia de acciones, sin que exista algún fin de negocios más que obtener un ahorro fiscal.

En el ejemplo propuesto, el reconocer la existencia de una relación mixta, que haga aplicable la CAG de manera subsidiaria, permitirá atribuir las consecuencias fiscales que resulten aplicables a la práctica elusiva desarrollada por las partes. Ello en tanto la estructura efectuada califica como fraude a la ley, por lo que, en consecuencia, procederá la recalificación de la transacción realizada como la transferencia de activo. No se considera que en el caso analizado pueda configurarse el concepto de economía de opción, pues existe un evidente ánimo elusivo, por parte de los sujetos intervinientes, para evitar una mayor carga impositiva de la que efectivamente le corresponde. Lo cual, a su vez, implica una contravención al principio de capacidad contributiva e igualdad.

Sobre la base de lo antes mencionado consideramos que, en lo que se refiere a la CAE regulada en el numeral 3 del artículo 105 de la LIR, debe reconocerse la existencia de una relación mixta con la CAG que haga factible al fisco someter a las transacciones que no cumplan con los parámetros de la CAE a un análisis, según las disposiciones de la CAG. Ello va a significar que el ente fiscal cuente con un mayor ámbito de acción para combatir el desarrollo de prácticas elusivas, como la descrita en el ejemplo previamente desarrollado.

\section{Conclusiones}

El fraude a la ley se constituye como una forma de elusión de la norma tributaria, para ello se 
utiliza una norma de cobertura que oculte el objetivo ulterior que es obtener un ahorro fiscal, al reducir o eliminar el impacto impositivo de una transacción.

La economía de opción se configura cuando un sujeto utiliza mecanismos legales que le permiten obtener un ahorro fiscal, sin incurrir en una trasgresión de la norma impositiva.

La CAG establece que una práctica será calificada como elusiva y, como consecuencia de ello, susceptible de recalificación, siempre que se superen los test de propiedad y de sustancia económica.

La CAG se sustenta en los principios de igualdad y de capacidad contributiva, en tanto estos implican el sometimiento equitativo de los contribuyentes a las cargas impositivas que les resulten atribuibles como consecuencia de la configuración de un determinado hecho imponible.

Las CAE son la respuesta normativa respecto al desarrollo de prácticas elusivas individualizadas por parte de los contribuyentes.

La CAE recogida en el numeral 3 del artículo 105 de la LIR se regula como una presunción absoluta, resultando aplicable en tanto se realice una escisión y se transfieran o cancelen las acciones adquiridas como consecuencia de dicha reorganización empresarial, conforme a los parámetros cuantitativos y temporales previstos en dicha norma.

En tanto la CAE prevista en el numeral 3 del artículo 105 de la LIR se regula como una presunción absoluta se debe reconocer que la relación con la CAG es mixta, y como consecuencia de ello, que resulta factible la aplicación de la CAG de manera subsidiaria, con el fin de permitir a la Administración Tributaria combatir de una manera más eficiente la elusión fiscal.

\section{Referencias bibliográficas}

Bravo Cucci, J. (2020). Elusión Tributaria. Lima: Instituto Pacífico.

Caliendo, P. (2018). Derecho Tributario y Análisis Económico del Derecho. Lima: Grijley.

Chumacero Quispe, R. (2018). Tras la búsqueda de una sana convivencia: Normas antielusivas generales y específicas en el derecho tributario peruano. En XIV Jornadas Nacionales de Derecho Tributario: La Cláusula Antielusiva General en el Ordenamiento Peruano (pág. 241). Lima: Instituto Peruano de Derecho Tributario.

Decreto Supremo N 145-2019-EF. (3 de mayo de 2019). Normas Legales, N 14929. Diario Oficial El Peruano, 6 de mayo de 2019. https://busquedas.elperuano.pe/normaslegales/aprueban-los-parametros-de-fondo-yforma-para-la-aplicacion-decreto-supremo-n-145-2019-ef-1766383-1/ 
Gamba Valega, C. (2014). Sobre el ITAN y el principio de capacidad económica. Una «conformidad» difícil de entender. En Investigaciones Tributarias y de Política Fiscal. Volumen I (pág. 188). Lima: Grijley.

Lizárraga Ibáñez, J. (2018). La Cláusula antielusiva general y su relación con las cláusulas antielusivas específicas. En XIV Jornadas Nacionales de Derecho Tributario: La Cláusula Antielusiva General en el Ordenamiento Peruano (págs. 468-469). Lima: Instituto Peruano de Derecho Tributario.

Medrano, H. (2018). Derecho tributario. Impuesto a la renta: aspectos significativos. Lima: Pontificia Universidad Católica del Perú.

Mur Valdivia, M. (2018). Cláusula General Antielusiva. Norma XVI del Título Preliminar del Código Tributario. En XIV Jornadas Nacionales de Derecho Tributario. La Cláusula Antielusiva General en el Ordenamiento Peruano (pág. 607). Lima: Instituto Peruano de Derecho Tributario.

Picón Gonzales, J. (2020). Paraísos Fiscales: Rompiendo mitos. Evolución, usos y medidas antiparaísos. Lima: Dogma Ediciones.

Queralt, J., Lozano, C. y Tejerizo, J. (2020). Curso de Derecho Financiero y Tributario. Madrid: Tecnos.

Saavedra Rodríguez, R. (2019). Reflexiones en torno al uso de presunciones legales en la determinación de la obligación tributaria. Volumen II. En Derecho Constitucional Tributario (pág. 1126). Lima: Legales Ediciones.

Sánchez Huete, M. Á. (2019). Tributación, fraude y blanqueo de capitales. Entre la prevención y la represión. Madrid: Marcial Pons.

Sentencia del Tribunal Constitucional 2727-2002-AA/TC, STC 2727-2002-AA/TC (Tribunal Constitucional, 2002).

Spisso, R. (2011). Derecho Constitucional Tributario. Buenos Aires: Abeledo Perrot.

Tarsitano, A. (2021). La elusión fiscal. Buenos Aires: Astrea.

Texto Único Ordenado de la Ley del Impuesto a la Renta. (2004). Decreto Supremo 179-2004-EF Texto Único Ordenado de la Ley del Impuesto a la Renta.

Texto Único Ordenado del Código Tributario aprobado mediante Decreto Supremo 133-2013-EF. (2013). Texto Único Ordenado del Código Tributario aprobado mediante Decreto Supremo 133-2013-EF. 